\title{
Health-friendly, high-quality white light using violet-green-red laser and InGaN nanowires-based true yellow nanowires light-emitting diodes
}

\author{
BILAL JANJUA ${ }^{\mathrm{a}}$, TIEN K. NG ${ }^{\mathrm{a}}$, CHAO ZHAO ${ }^{\mathrm{a}}$, DALAVER H. ANJUM ${ }^{\mathrm{b}}$, ADITYA \\ PRABASWARA $^{\mathrm{a}}$, GIUSEPPE BERNARDO CONSIGLIO ${ }^{\mathrm{a}}, \mathrm{CHAO}^{\mathrm{SHEN}}{ }^{\mathrm{a}}$ AND \\ BOON S. OOI ${ }^{\mathrm{a}^{*}}$ \\ ${ }^{a}$ King Abdullah University of Science and Technology (KAUST), Photonics Laboratory, Thuwal \\ 23955-6900, Saudi Arabia; ${ }^{\mathrm{b}}$ King Abdullah University of Science and Technology (KAUST), \\ Imaging and Characterization Core Lab, Thuwal 23955-6900, Saudi Arabia. \\ *corresponding author: boon.ooi@kaust.edu.sa
}

\begin{abstract}
White light based on blue laser - YAG: $\mathrm{Ce}^{3+}$ phosphor has the advantage of implementing solid-state lighting and optical wireless communications combined-functionalities in a single lamp. However, the blue light was found to disrupt melatonin production, and therefore the human circadian rhythm in general; while the yellow phosphor is susceptible to degradation by laser irradiation and also lack tunability in color rendering index (CRI). In this investigation, by using a violet laser, which has $50 \%$ less impact on circadian response, as compared to blue light, and an InGaN-quantum-disks nanowires-based light-emitting diode (NWs-LED), we address both issues simultaneously. The white light is therefore generated using violet-green-red lasers, in conjunction with a yellow NWs-LED realized using molecular beam epitaxy technique, on titanium-coated silicon substrates. Unlike the conventional quantum-well-based LED, the NWs-LED showed efficiency-droop free behavior up to $9.8 \mathrm{~A} / \mathrm{cm}^{2}$ with peak output power of $400 \mu \mathrm{W}$. A low turn-on voltage of $\sim 2.1 \mathrm{~V}$ was attributed to the formation of conducting titanium nitride layer at NWs nucleation site and improved fabrication process in the presence of relatively uniform height distribution. The $3 \mathrm{D}$ quantum confinement and the reduced band bending improve carriers-wavefunctions overlap, resulting in an IQE of $\sim 39 \%$. By changing the relative intensities of the individual color components, CRI of $>85$ was achieved with tunable correlated color temperature (CCT), thus covering the desired room lighting conditions. Our architecture provides important considerations in designing smart solid-state lighting while addressing the harmful effect of blue light.
\end{abstract}

Keywords: Yellow, nanowire, light emitting diode, laser, violet, white light, solid-state lighting, circadian rhythm.

\section{INTRODUCTION}

In the past decade, solid state lighting (SLL) based on light emitting diodes (LEDs) and laser diodes (LDs) have gained significant attractiveness. Use of an environmental friendly nitride material in realizing highly efficient and stable sources have been the main driving force behind the SSL technology. Still, bottlenecks such as large dislocation density and strong polarization fields of several MV. $\mathrm{cm}^{-1}$ results in reduced electron-hole wavefunction overlap [1]. Also when targeting wavelength above green color severe alloy clustering and strain-induced defects also result in poor crystalline quality. Planar devices with high indium composition have been demonstrated, but they suffer from low IQE as well as pronounced 'efficiency-droop' which is a decrease in external quantum efficiency (EQE) with an increase in injection current.

Unlike planar semiconductor epitaxy growth; self-assembled catalyst-free nanowires (NWs) materials can be grown on cheap scalable substrates with ease. Since the NWs are grown via radial strain relaxation, the condition for lattice matched substrates is relaxed. This allows epitaxial growth of long single crystal NWs, free of dislocation; which remains a challenge in realizing efficient planar devices. The NWs can be grown at a low temperature in the presence of kinetically driven plasma thus allowing effective incorporation of indium for higher wavelength light emitting devices. In the presence of reduced strain, these structures exhibit weaker piezopolarization fields allowing the carriers to recombine with shorter lifetimes. Using these inherent advantages of NWs, optical devices in the visible regime have 
been demonstrated albeit with shortcomings such as poor thermal dissipation, higher turn voltage, and Si absorption losses $[2,3]$. Formation of unwanted $\mathrm{Si}_{3} \mathrm{~N}_{4}$ at the NW/Si interface has been identified as the main culprit. $\mathrm{Si}_{3} \mathrm{~N}_{4}$ being an insulator facilitate lateral confinement of acoustic phonons that are restricted to one-dimension transport thus resulting in severe junction heating [4-7]. Even then, superior emission properties of NWs have resulted in the demonstration of lasers having emission as far as $1.2 \mathrm{um}$ using $\mathrm{InGaN}$ as an active medium [8].

For conventional white light generation mixing of blue and a broad yellow component of the visible spectrum is required. Typically the conformal YAG: $\mathrm{Ce}^{3+}$ coating absorbs the blue light and reemits a broad yellow spectrum $[9,10]$. Even though this technology has the potential to produce white light with high theoretical efficiency the passive yellow phosphor components lacks tunability, low efficiency due to down conversion and temporal material degradation under high power operation [11-13]. Also the blue light, an important component in the solid-state-lightning technology, has been shown to have a negative impact on health. Though exposure to blue light during the daytime helps boost alertness; however prolonged exposure at night has been hypothesized to affect circadian rhythm resulting in disruption of sleep schedule. The Melanopsin-based photoreceptor most likely cause of such behavior under blue light exposure is linked to decreasing in the body production of melatonin [14] [15]. Studies have shown that the action spectra for melatonin suppression peaks around $460 \mathrm{~nm}$ and decreases by $50 \%$ around $400 \mathrm{~nm}[16,17]$. For improved sleep, it has been recommended by health experts to reduce blue light intensity at night [18]. Therefore there is a need for an alternative health-friendly reliable solution for achieving high-quality white lightening.

One such approach is to use a solid-state yellow LED as an active phosphor in conjunction with a red, green and violet lasers to produce white light with excellent characteristics. A similar concept has been demonstrated in our previous work by employing an orange LED in union with red, green and blue LDs [19]. For the yellow component with required emission around $580-590 \mathrm{~nm}$, NWs based optical device is the best candidate. Growing such structures on metal-coated Si provides the motivation of avoiding $\mathrm{Si}_{3} \mathrm{~N}_{4}$ while also providing a reflective based configuration for color mixing. In addition, NWs based devices have shown to have the much needed broader emission spectrum for it to replace YAG: $\mathrm{Ce}^{3+}$ based phosphor effectively.

Here we report a white light generation using RGV LDs and broad linewidth, true yellow InGaN quantum-disks (Qdisks)-in-NWs LED operating at room temperature. The peak emission of the yellow LED of $589 \mathrm{~nm}$ was obtained at $9.8 \mathrm{~A} / \mathrm{cm}^{2}$ bias $\left(98 \mathrm{~mA}\right.$ over an area of $\left.1 \times 1 \mathrm{~mm}^{2}\right)$ with an FWHM of $\sim 73.88 \mathrm{~nm}$. The LED showed a low turn-on voltage of $2.1 \mathrm{~V}$. The absolute peak optical power measured was $400 \mu \mathrm{W}$ at $9.8 \mathrm{~A} / \mathrm{cm}^{2}$ with an IQE of $\sim 39 \%$. By implementing the true yellow NWs LED as a source of yellow phosphor light, and as a light scattering plane, in a reflective configuration, for color-mixing with red, green and violet (RGB) laser photons, we present a novel solution to phosphor/LD architecture, which allows tunability while showing excellent white color quality. Our lighting architecture considers the exclusion of blue light, in significantly removing its detrimental effect to the human circadian rhythm, in designing smart solid-state lighting.

\section{Experimental}


Figure 1. Schematic of the white light generation experiment using yellow LED and red, green and violet (RGV) LDs. (a) Probed yellow NWs LED. (b) Image of white light generated in a reflective configuration 
For white light generation, red (LP642-SF20), green (LP520-SF15), and violet (LP405-SF10) LDs from Thorlabs, with nominal spectral linewidth / center wavelength of $1 \mathrm{~nm} / 640 \mathrm{~nm}, 0.48 \mathrm{~nm} / 515 \mathrm{~nm}, 1.1 \mathrm{~nm} / 405 \mathrm{~nm}$, respectively. The white light experiment was performed using collimated laser beams focused onto the NWs samples using plano-convex optics. The diameter of the spots was adjusted to the scale of the device $\left(1 \times 1 \mathrm{~mm}^{2}\right)$. The laser-beams were incident at an angle to the device surface to extend laser-beam spot and increase their interaction with the NWs. This, in turn, produces a projection of white light on a screen at $50 \mathrm{~mm}$ away, and normal to the yellow LED sample. The mixed white light was measured using a GL Opti-probe attachment, fiber-coupled into the GL Spectis 5.0 Touch spectrometer as shown in Figure 1(a)-(c).

The yellow LED was grown on $100 \mathrm{~nm}$ Ti coated Si substrate using plasma-assisted molecular beam epitaxy. NWs were first nucleated at $500{ }^{\circ} \mathrm{C}$ followed by high-temperature growth to improve crystal quality. Following Si-doped GaN growth five InGaN quantum disks were grown at $515{ }^{\circ} \mathrm{C}$ and were embedded in $\mathrm{GaN}$ matrix realized at $565{ }^{\circ} \mathrm{C}$. Beam equivalent pressure measured by beam flux monitor was $5 \times 10^{-8}$ Torr and 3-6 $\times 10^{-8}$ Torr for indium and gallium. Metal to nitrogen fluxes ratio was kept at approximately one to three to prompt three-dimensional vertical growth of NWs.

The NWs were characterized, following growth, using FEI Quanta 3D FEG field emission electron microscopy. For material quality high-resolution TEM was used to examine NWs in the [0002] direction. Emission from the active region was measured using a micro-PL setup with $405 \mathrm{~nm}$ excitation source and Ocean Optics QE6500 spectrometer. McScience LC50000 LED tester was utilized to measure the current-voltage-power characteristics of the devices and the respective electroluminescence spectrum.

The yellow LED was fabricated using standard UV lithography methods. First, the NWs were planarized using parylene followed by $\mathrm{Ni}(5 \mathrm{~nm}) / \mathrm{Au}(5 \mathrm{~nm})$ deposition and annealing to form an ohmic contact. Thick Au was then deposited at the top and bottom of the wafer to define contacts.

\section{Results and Discussion}
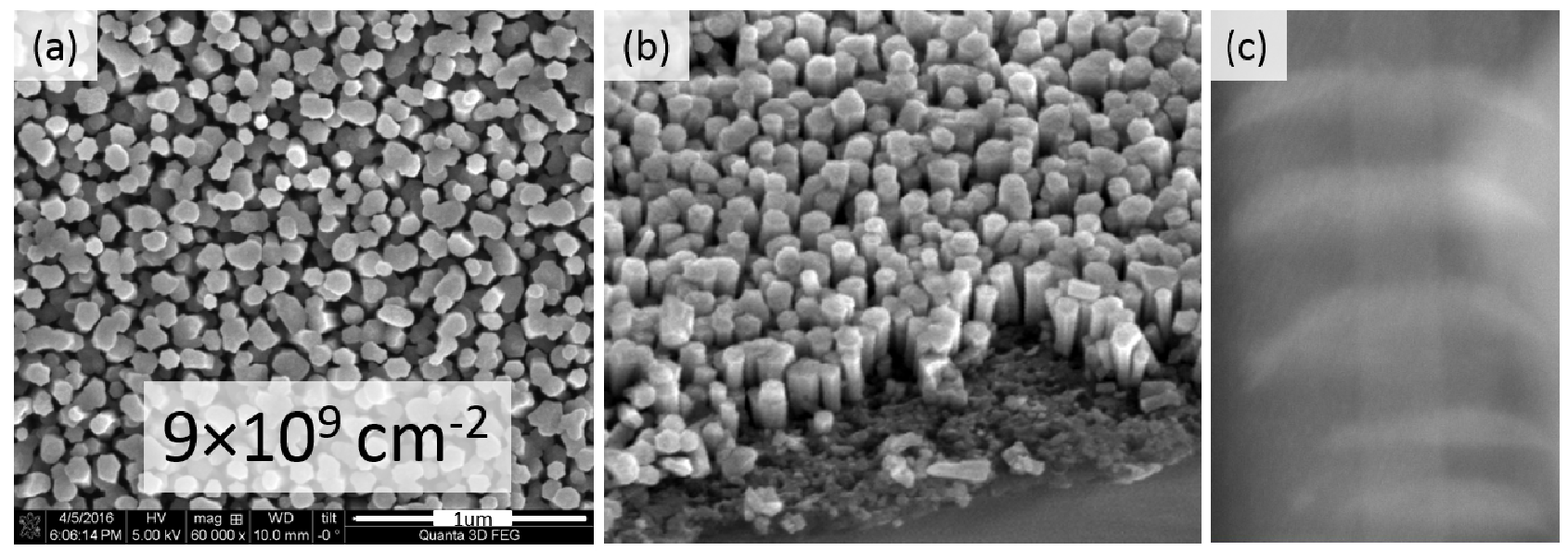

Figure 2. (a) Top view SEM image of the device sample. (b) Elevation view shows disjointed vertical NWs. (c) A TEM image of the active region shows truncated hexagonal InGaN Qdisks in GaN matrix.

Figure 2(a) shows the top view SEM image of the NWs device sample. An average lateral size and length were measured to be $55 \mathrm{~nm}$ and $200 \mathrm{~nm}$. The NWs were most vertically aligned and disjointed with a fill factor of more than $80 \%$ and approximate density of $9 \times 10^{9} \mathrm{~cm}^{-2}$ as shown in Fig. 2(b). The NWs have a tapered shape with the minimum lateral diameter at its base. TEM image in Fig. 2 (c) shows clearly formation of the InGaN Qdisks in GaN matrix. Such shape of the NWs has been reported to form under built-in strain and inhomogeneous surface temperature variation across the length of the NWs $[2,20]$. Gallium having a smaller diffusion length tends to get incorporated to the sidewall resulting in a spontaneous formation of encapsulation around the InGaN Qdisks. 
For the white light experiment, a broad linewidth spectrum improves the light quality. As shown in Fig. 3(a) the device structure utilized incorporates InGaN Qdisks to generate a broad emission. To study its optical properties, room temperature photoluminescence $(\mathrm{PL})$ is performed.
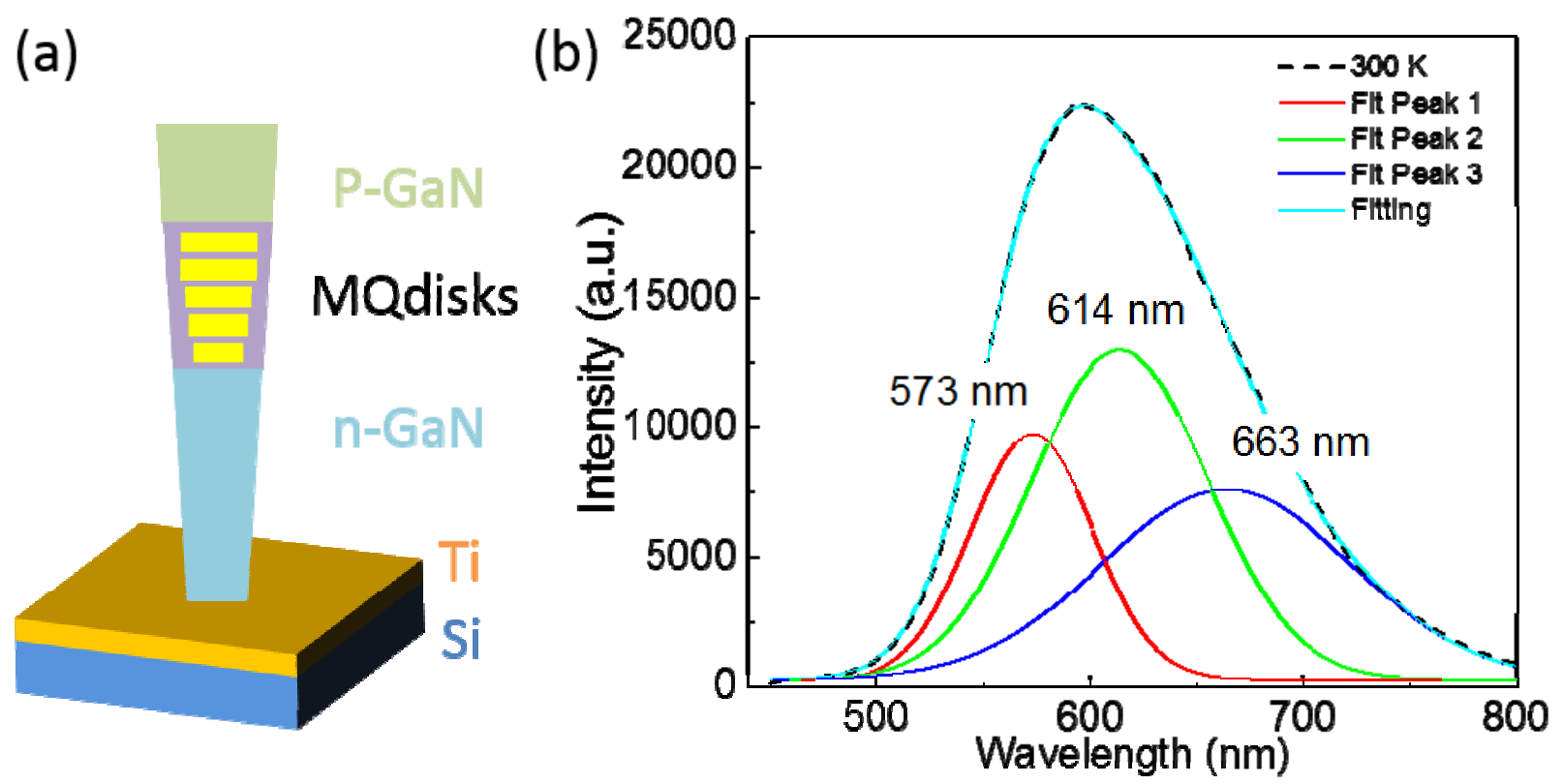

Figure 3. (a) Schematic of the NW with individual layers highlighted. (b) Photoluminescence spectrum of the NWs at room temperature being fitted with three Gaussian peaks.

A broad linewidth of $130 \mathrm{~nm}$ with a peak at $595 \mathrm{~nm}$ is obtained. The broadening can be due to the presence of the compositional inhomogeneity across a Qdisk due to alloy clustering and across the active region due to temperature and strain variation. Since the NWs nucleation process is governed thermodynamically, variation in diameter and length also leads to the broadening effect in the PL intensity. In Fig 3(b) the spectrum is fitted with three Gaussian peaks. Peak 2 correlates well with electroluminescence showing emission from the active region. Appearance of peak 1 can be linked to an excitation of a different ensemble of NWs and peak 3 to band tail effect and inhomogeneity. IQE based on lowtemperature PL was measured to be around $40 \%$ [21].
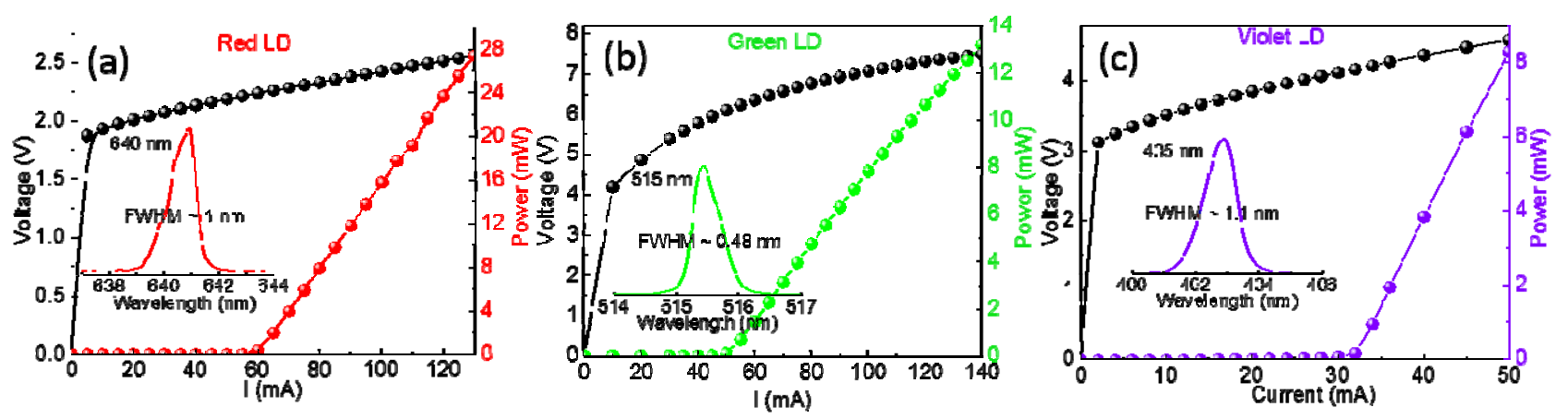

Figure 4. Optical and L-I-V characteristics of (a) red, (b) green and (c) violet laser diode.

Sufficient characterization of the LDs was being done prior to the white light experiment. Figure 4(a)-(c) shows the light output power-current-voltage (LIV) curves of the RGV LDs having a threshold current of $\sim 60 \mathrm{~mA}, \sim 51$ and ??. The lasing wavelength and FWHM of the RGV LDs are 640/1 nm, 515/0.48 nm and 405/1.1 nm as shown in insets. 

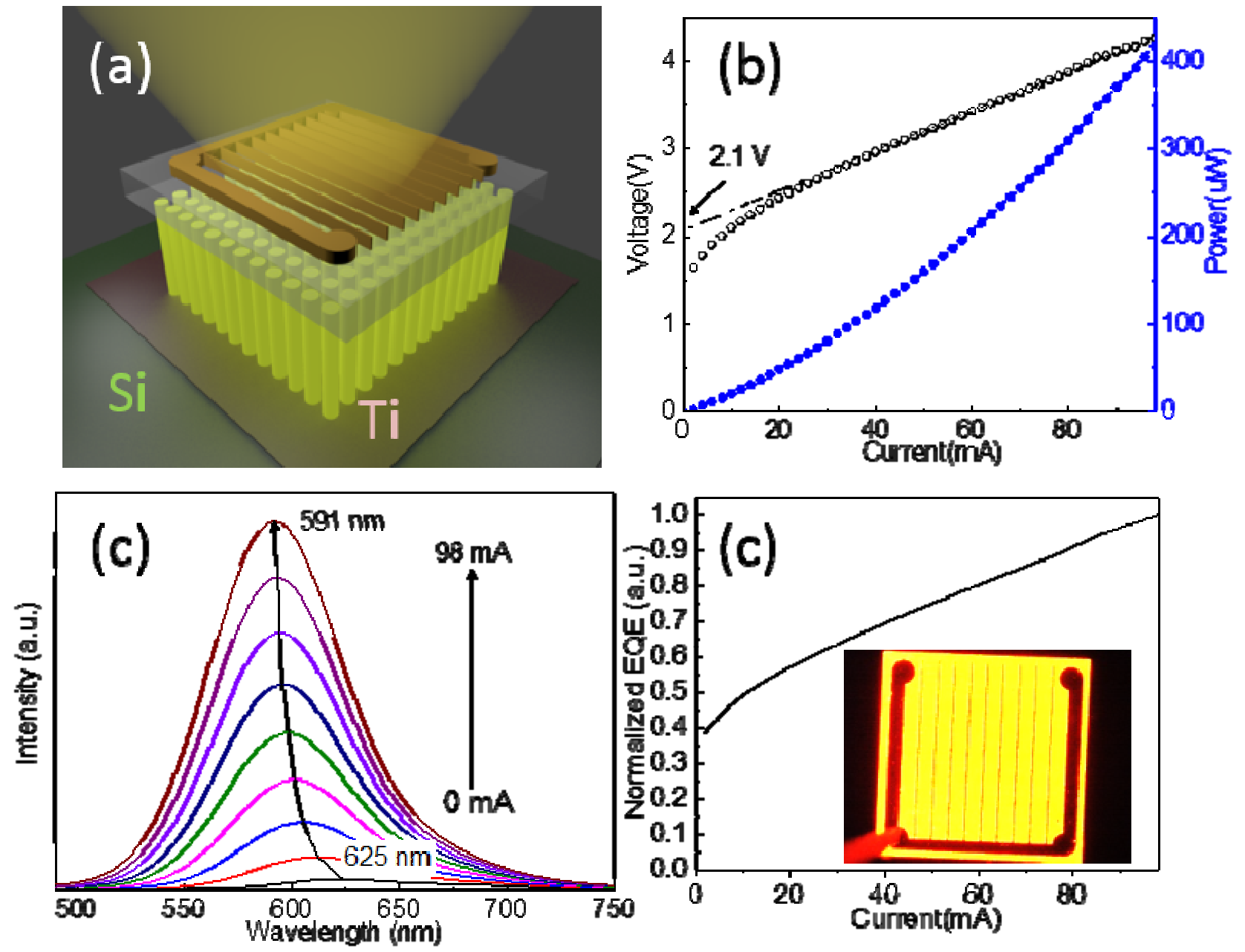

Figure 5. (a) 3D schematic of the fabricated NWs device with top contacts and underlying Ti coated Si substrate. (b) L-I-V plot of the $1 \times 1 \mathrm{~cm}^{2}$ yellow NWs LED. EL plot of the device shows emission of $591 \mathrm{~nm}$ at $9.8 \mathrm{~A} / \mathrm{cm}^{2}$ of injection current. (d) EQE plotted against wavelength shows droop-free behavior up to injection current of $9.8 \mathrm{~mA} / \mathrm{cm}^{2}$.

Figure 5(a) shows the 3D depiction of the fabricated NWs yellow LED. The underlying Ti layer helps to reflect the impinging light. A low turn-on voltage of $2.1 \mathrm{~V}$ was obtained with droop-free behavior up to $9.8 \mathrm{~A} / \mathrm{cm}^{2}$. The peak emission of $591 \mathrm{~nm}$, having a linewidth of $74.7 \mathrm{~nm}$, was measured at $9.8 \mathrm{~A} / \mathrm{cm}^{2}$. The EL spectrum shows a blue shift of $34 \mathrm{~nm}$ with variation in injection current from $0-9.8 \mathrm{~A} / \mathrm{cm}^{2}$ as shown in Fig. 5 (c). The shift is attributed to preferential pumping of NWs ensemble emitting in yellow. Quantum-confined stark effect has been ruled out as these structures are observed to be strain free with reduced piezo polarization fields. The PL and EL being two different processes show good consistency. A homogenous light emission from the $1 \times 1 \mathrm{~mm}^{2}$ device is shown as an inset in Fig.5 (c),confirming suitable fabrication process. The measured correlated color temperature (CCT) and color rendering index (CRI) values of the standalone yellow NWs LED was $2382 \mathrm{~K}$ and 39.3.

CCT is a measure of light source color appearance in comparison to a black body radiator. CCT is given in Kelvin with values above $5000 \mathrm{~K}$ are considered as cool colors and below, warm colors. While CRI provides an indication of the ability of the light source to reveal the color of various objects in comparison to response to an ideal source. CRI are unitless values with maximum 100 given to a black body radiator. 


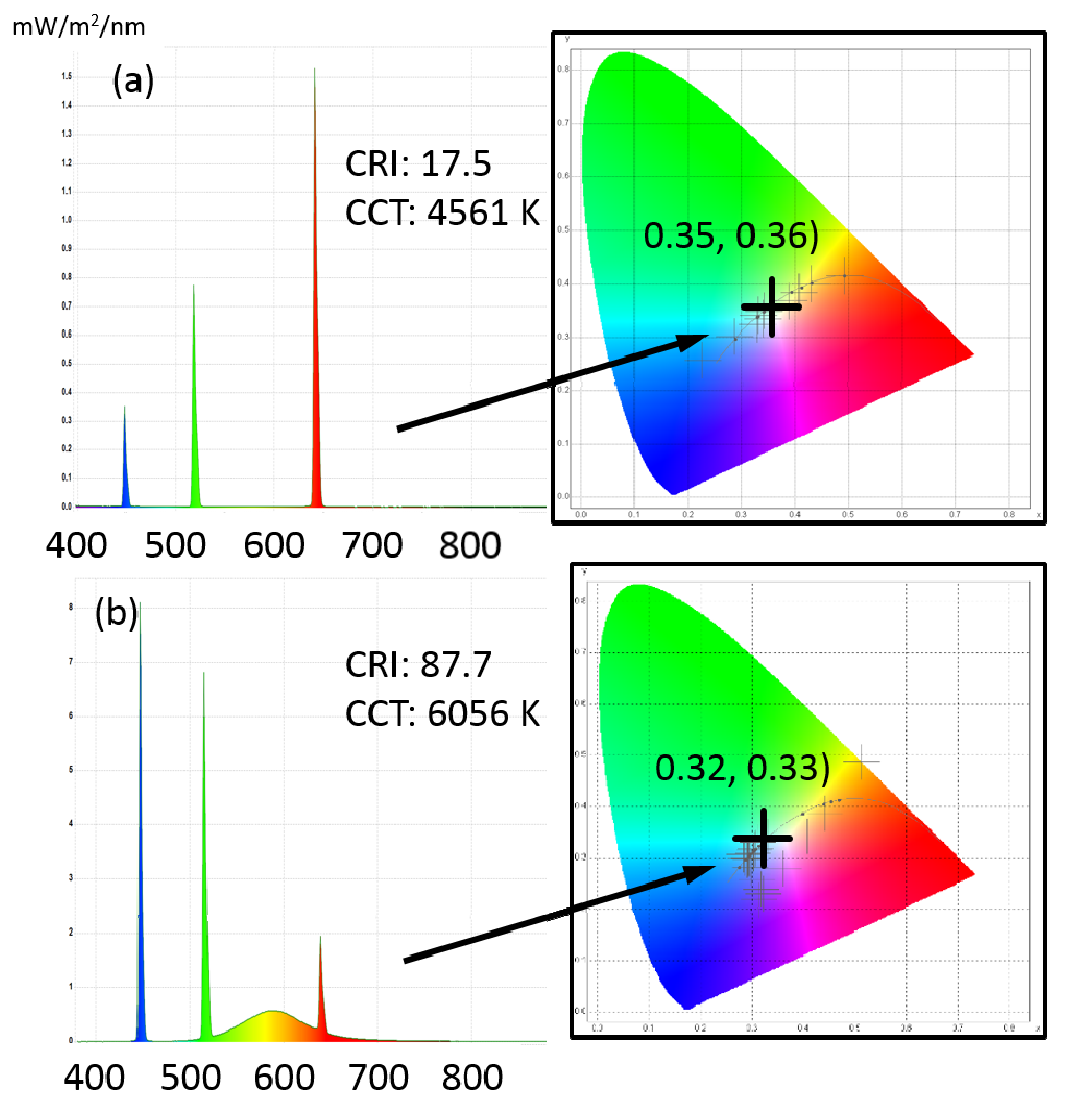

Figure 6. (a) The white light spectra generated using RGB LD (b), and RGB LD in conjunction with yellow NWs LED selected from

those showing the best CRI values of 17.5 and 87.7, respectively, with the respective color coordinates and CCT values indicated.

Systematically characterization of white light using RGB LDs based on CCT values has been done in our previous work $[22,23]$. As can be seen in Fig.6(a), with RGB LDs, the Planckian locus line depicting white light with different CCT values can be covered. This is possible by individually changing the LDs intensities with respect to each other. The drawback of relying on RGB LDs is that the quality of white light, as quantified by CRI value, is considerably lower in the absence of broad linewidth light signals; and thus prevent this technology to be of any practical use. Though red, green and blue LED with relatively broader linewidth have shown tunable CCT with high CRI values, smaller bandwidth and 'efficiency droop' issue have limited their use in high-speed communication and high power operation applications [24]. Tsao et al. in his recent work showed significantly improved white light quality by introducing a yellow LD in the RGB configuration [23]. The yellow component was obtained using frequency doubling method which can reduce the cost effectiveness and stability of the system. In our recent work, we demonstrated NWs based yellow LED grown on cheap Si substrate to achieve good white light characteristics while also maintaining the niche of color tunability. As shown in Fig. 5(b) the best CRI obtained was 87.7 with a CCT of $6056 \mathrm{~K}$ [21]. Such high CRI values are comparable to that obtained for commercially used incandescent lamps.

Though with blue light, high-quality white light can be obtained, the health related issues provide the motivation to adopt shorter wavelengths as a substitute. In this regard, an obvious choice, at night, is to devices emitting in the violet regime in mediating human circadian rhythms. In our experimental, we used a violet laser, emitting at $405 \mathrm{~nm}$, as a possible replacement for blue LD. 


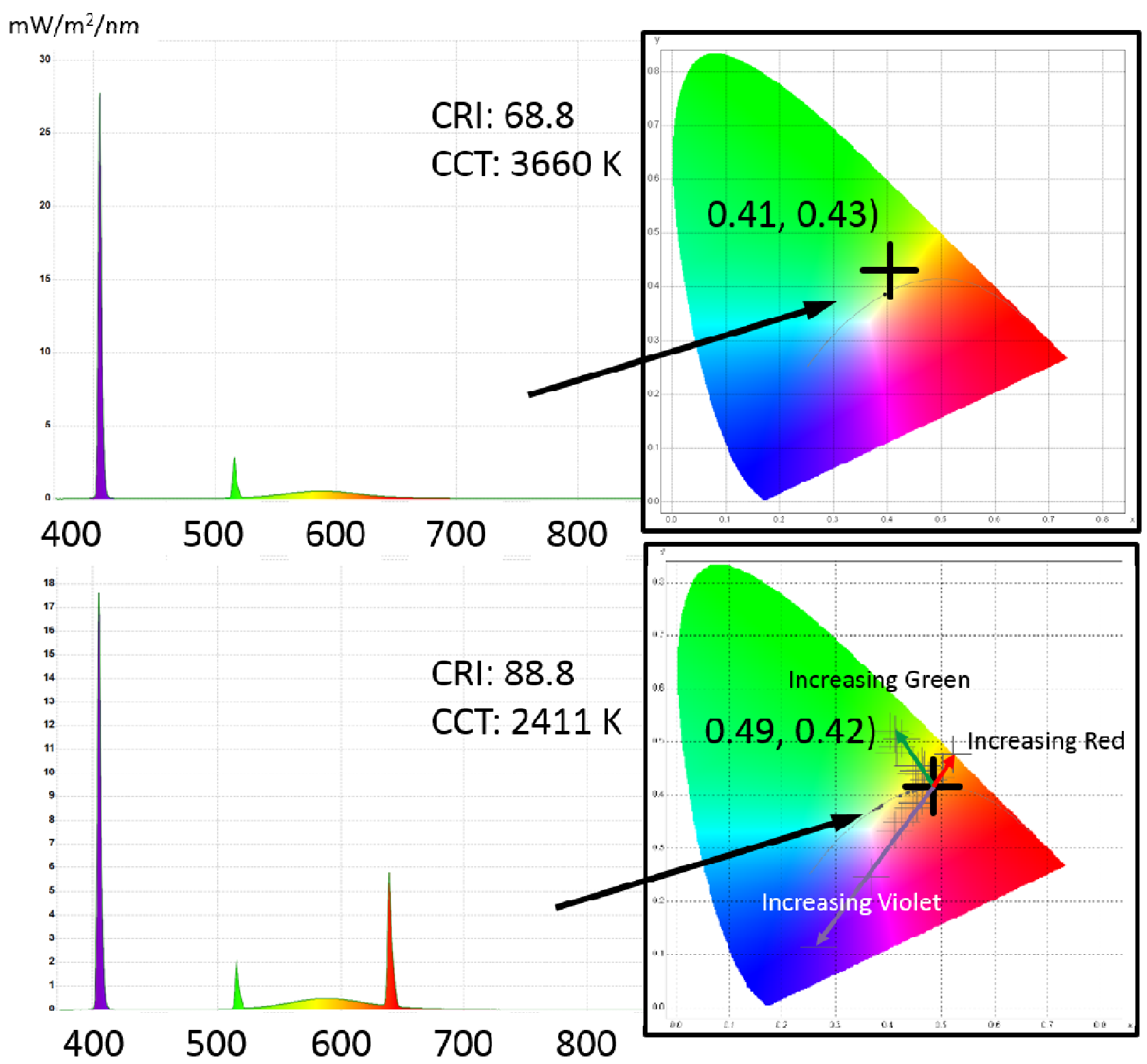

Figure 7. (a) The white light spectra generated using GV LD and (b), and RGV LD in conjunction with yellow NWs LED selected from those showing the best CRI values of 68.8 and 88.8 , respectively, with the respective color coordinates and CCT values indicated.

As can be seen in Fig.7(a ) with the green violet (GV) LD and yellow LED, CRI above 65.5 was obtained with a CCT value of $3660 \mathrm{~K}$. Such CRI values can be seen in single LD and YAG phosphor based solution [25]. Since for indoor lighting, it is desired to have CRI values above 80, we incorporated a red LD, in our setup. With the new configuration of red, green and violet (RGV) LD and yellow LED we were able to push the CRI above 85 having CCT temperature of $2411 \mathrm{~K}$. It was observed, in comparison to RGB LD and yellow LED which shows cooler white light characteristics for high CRI values, violet based configuration gives much warmer white light as stated in Table. 1. In another study, the blue light component was significantly reduced in conjunction with violet LD while having CRI values above 80 . Figure 7(b) inset shows with drawn arrow how the coordinates of the white light change on the color chromaticity space. With increase in violet LD intensity the white color light shows cooler characteristics. Similar trend is observed with red LD which shows an increase in CCT value with increase in intensity. Though the CRI values decrease from the optimum 
value indicated with black arrow, further tweaking of the LD intensities can re-attain good white light characteristic values.

Table 1. Measured CCT and CRI values for different combinations of RGV LDs and yellow LED intensities.

\begin{tabular}{|c|c|}
\hline CCT (K) & CRI \\
\hline 4215 & 65.3 \\
\hline 4067 & 66.6 \\
\hline 3908 & 69.3 \\
\hline 3211 & 77.5 \\
\hline 3022 & 80 \\
\hline 2819 & 82.7 \\
\hline 2610 & 86.1 \\
\hline 2411 & 88.8 \\
\hline
\end{tabular}

\section{Summary}

GaN-based laser light emitting devices in lighting and visible light communication have attracted considerable attention because of their stability, efficient power consumption, and high-speed modulation capability. However, the blue component in the blue LDs/yellow-emitting YAG: $\mathrm{Ce}^{3+}$ phosphor white light generation platform have health related issues. By making use of the true yellow NWs LED in conjunction with red, green and violet LDs, we present a new solution to active-phosphor/LD architecture, which allows tunability while showing excellent color quality. We were able to get a warm white light with CCT of $2411 \mathrm{~K}$ and high CRI 88.8. Such results are comparable to more conventional blue LD based white light solutions. Our lighting architecture considers the removal of blue light, in significantly removing its detrimental effect to the human circadian rhythm, in designing smart solid-state lighting.

\section{References}

[1] H. P. Zhao, G. Y. Liu, J. Zhang et al., "Approaches for high internal quantum efficiency green InGaN light-emitting diodes with large overlap quantum wells," Optics Express, 19(14), A991-A1007 (2011).

[2] C. Zhao, T. K. Ng, N. N. Wei et al., "Facile Formation of High-Quality InGaN/GaN Quantum-Disks-in-Nanowires on Bulk-Metal Substrates for High-Power Light-Emitters," Nano Letters, 16(2), 1056-1063 (2016).

[3] H. P. T. Nguyen, S. Zhang, K. Cui et al., "p-Type Modulation Doped InGaN/GaN Dot-in-aWire White-Light-Emitting Diodes Monolithically Grown on Si(111)," Nano Letters, 11(5), 1919-1924 (2011).

[4] J. Matthews, E. A. Hoffmann, C. Weber et al., "Heat flow in InAs/InP heterostructure nanowires," Physical Review B, 86(17), (2012).

[5] J. H. Cheng, D. Seghete, S. M. George et al., "Thermal Management of Vertical Gallium Nitride Nanowire Arrays: Cooling Design and Tip Temperature Measurement,” Mems 2010: 23rd Ieee International Conference on Micro Electro Mechanical Systems, Technical Digest, 468-471 (2010). 
[6] Q. M. Li, K. R. Westlake, M. H. Crawford et al., "Optical performance of top-down fabricated InGaN/GaN nanorod light emitting diode arrays," Optics Express, 19(25), 2552825534 (2011).

[7] C. Zhao, T. K. Ng, A. Prabaswara et al., "An enhanced surface passivation effect in InGaN/GaN disk-in-nanowire light emitting diodes for mitigating Shockley-Read-Hall recombination," Nanoscale, 7(40), 16658-16665 (2015).

[8] A. Hazari, A. Aiello, T. K. Ng et al., "III-nitride disk-in-nanowire $1.2 \mathrm{mu}$ m monolithic diode laser on (001) silicon," Applied Physics Letters, 107(19), (2015).

[9] K. T. Bicanic, X. Li, R. P. Sabatini et al., "Design of phosphor white light systems for high power applications," ACS Photonics, (2016).

[10] J. R. D. Retamal, H. M. Oubei, B. Janjua et al., "4-Gbit/s visible light communication link based on 16-QAM OFDM transmission over remote phosphor-film converted white light by using blue laser diode," Optics express, 23(26), 33656-33666 (2015).

[11] Y. Ohno, "Color rendering and luminous efficacy of white LED spectra," Fourth International Conference on Solid State Lighting, 5530, 88-98 (2004).

[12] N. Narendran, Y. Gu, J. Freyssinier et al., "Solid-state lighting: failure analysis of white LEDs," Journal of Crystal Growth, 268(3), 449-456 (2004).

[13] I. Dursun, C. Shen, M. R. Parida et al., "Perovskite Nanocrystals as a Color Converter for Visible Light Communication," ACS Photonics, (2016).

[14] K. J. Navara, and R. J. Nelson, "The dark side of light at night: physiological, epidemiological, and ecological consequences," Journal of pineal research, 43(3), 215-224 (2007).

[15] F. Falchi, P. Cinzano, C. D. Elvidge et al., "Limiting the impact of light pollution on human health, environment and stellar visibility," Journal of environmental management, 92(10), 2714-2722 (2011).

[16] G. C. Brainard, J. P. Hanifin, J. M. Greeson et al., "Action spectrum for melatonin regulation in humans: evidence for a novel circadian photoreceptor," The Journal of Neuroscience, 21(16), 6405-6412 (2001).

[17] J. E. Roberts, "Circadian rhythm and human health," Department of Natural Sciences, Room, 813, (2010).

[18] A.-M. Chang, D. Aeschbach, J. F. Duffy et al., "Evening use of light-emitting eReaders negatively affects sleep, circadian timing, and next-morning alertness," Proceedings of the National Academy of Sciences, 112(4), 1232-1237 (2015).

[19] B. Janjua, T. K. Ng, C. Zhao et al., "Ultrabroad linewidth orange-emitting nanowires LED for high CRI laser-based white lighting and gigahertz communications," Optics Express, 24(17), 19228-19236 (2016).

[20] H. P. T. Nguyen, M. Djavid, S. Y. Woo et al., "Engineering the Carrier Dynamics of InGaN Nanowire White Light-Emitting Diodes by Distributed p-AlGaN Electron Blocking Layers," Scientific Reports, 5, (2015).

[21] B. Janjua, T. K. Ng, C. Zhao et al., "True Yellow Light-Emitting Diodes as Phosphor for Tunable Color-Rendering Index Laser-Based White Light," Acs Photonics, 3(11), 2089-2095 (2016).

[22] B. Janjua, H. M. Oubei, J. R. D. Retamal et al., "Going beyond 4 Gbps data rate by employing RGB laser diodes for visible light communication," Optics Express, 23(14), 18746-18753 (2015). 
[23] D. Tsonev, S. Videv, and H. Haas, "Towards a $100 \mathrm{~Gb} / \mathrm{s}$ visible light wireless access network," Optics Express, 23(2), 1627-1637 (2015).

[24] S. Muthu, F. J. Schuurmans, and M. D. Pashley, "Red, green, and blue LEDs for white light illumination," IEEE Journal of selected topics in quantum electronics, 8(2), 333-338 (2002).

[25] C. Lee, C. Shen, H. M. Oubei et al., " 2 Gbit/s data transmission from an unfiltered laserbased phosphor-converted white lighting communication system," Optics express, 23(23), 29779-29787 (2015). 